\title{
An elusive case of acute abdomen
}

\author{
Lt Col Vivek Aggarwal*, Lt Col Deepak Dwivedi ${ }^{\dagger}$, Col AV Akulwar ${ }^{\#}$
}

MJAFI 2011;67:380-381

\section{INTRODUCTION}

Acute abdomen is a very common surgical emergency and requires accurate history, clinical examination, and supporting investigation for diagnosis. Medical causes of acute abdomen are very rare and require a high index of suspicion to make the diagnosis. We are presenting one such case of recurrent acute abdomen due to a medical cause.

\section{CASE HISTORY}

A 26-year-old soldier with two years history of recurrent pain in the abdomen was admitted to our hospital on 2 November 2009 with fever, pain in abdomen, and vomiting of three days duration. Perusal of old documents revealed that he had been evaluated at tertiary care centres repeatedly for last two years. All the investigations including contrast-enhanced computed tomography (CECT) abdomen, colonoscopy, gastrointestinal (GI) endoscopy, enterocolysis were normal. As per the history available, individual had recurrent episodes of pain in abdomen with obstipation. There was one episode of altered sensorium associated with pain in abdomen two years ago. After that individual was labelled to be having somatic problems for which he was referred for psychiatric evaluation.

Present episode started on 29 October 2009 with moderate grade intermittent fever, vomiting, and mild periumbilical pain. Pain in abdomen increased on the second day of admission and became very severe with obstipation, thought to be due to subacute intestinal obstruction. The clinical examination found the abdomen to be soft and bowel sounds were present. Computerised tomography of the abdomen revealed mild hepatosplenomegaly. The patient responded to conservative management by keeping him nil by mouth, and administering IV fluids and cephalosporin antibiotics. Biochemical investigations revealed raised serum bilirubin $(2.2 \mathrm{mg} \%)$ with mildly raised transaminases. Serological test for malaria was negative; HBsAg (hepatitis B surface antigen) and anti-HCV

\footnotetext{
*Graded Specialist (Medicine), Military Hospital, Ramgarh Cantt., ${ }^{\dagger}$ Graded Specialist (Anaesthesia), Military Hospital, Bareilly, \#Senior Advisor (Surgery), 167 Military Hospital, C/o 56 APO.

Correspondence: Lt Col Vivek Aggarwal, Graded Specialist (Medicine), Military Hospital, Ramgarh Cantt.

E-mail:viksl20@yahoo.com
}

Received: 20.03.2010; Accepted: 11.07.2011

doi: 10.1016/S0377-1237(11)60092-0 (hepatitis $\mathrm{C}$ virus) were negative and subsequent liver function tests were within normal limits.

The patient had a recurrence of severe pain in abdomen again on 11 November 2009 along with tachycardia and raised blood pressure ranging between 150/100 and 160/110 mmHg. The clinical examination found the abdomen to be soft without rigidity and guarding. Radiography and ultrasonography of the abdomen were found to be normal. The pain in the abdomen responded poorly to analgesics. The patient started having abnormal behaviour on 13 November 2009. All haematological and biochemical investigations, including serum electrolytes done at this hospital were normal. At this juncture, the patient was suspected to have acute intermittent porphyria (AIP); however, the urine colour did not change on keeping in sunlight. Urine sample was taken again for testing for porphobilonogen and was sent to a civil laboratory in Mumbai.

On 14 November 2009, the patient had altered sensorium with one episode of generalised tonic clonic seizure (GTCS). Serum electrolytes done from a civil laboratory showed severe hyponatraemia with sodium levels $103 \mathrm{mmol} / \mathrm{L}$. However, potassium and magnesium were normal. The patient was started on $3 \% \mathrm{NaCl}$ drip at the rate of $35 \mathrm{~mL} /$ hour for correction. But, the patient was hyperventilating and developed carpopedal spasm, so IV calcium gluconate was administered. Arterial blood gas (ABG) analysis showed picture of respiratory alkalosis. The patient was put on mechanical ventilator for airway protection as patient was stuprose with Glasgow coma scale (GCS) of $8 / 15$ due to severe hyponatraemia and for correction of respiratory alkalosis and severe respiratory fatigue which patient developed. At this point the urine collected was dark brown in colour (Figure 1), urine R/E was normal, urinary sodium was $163 \mathrm{mmol} / \mathrm{L}$, and he was diagnosed as a suspected case of AIP

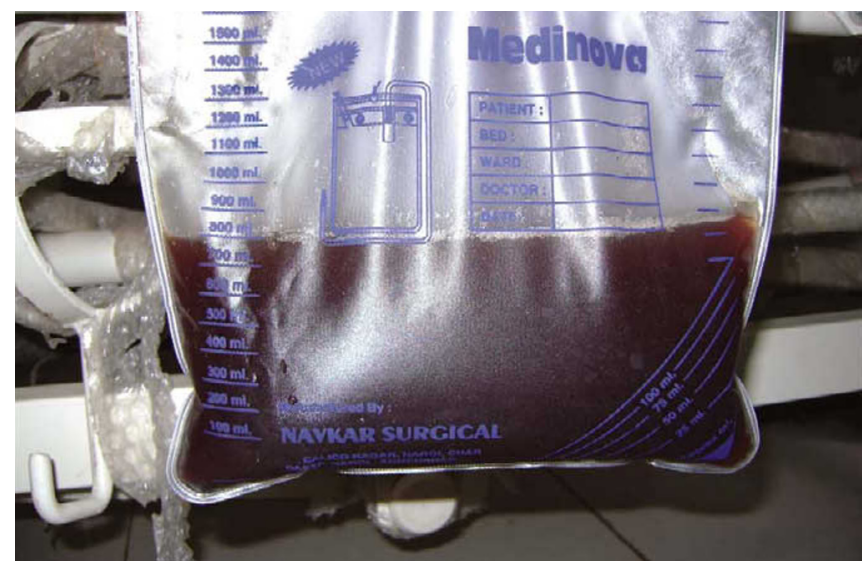

Figure 1 Colour of the urine during acute porphyric crisis. 
Table 1 Serial sodium levels.

$\begin{array}{lccc}\text { S. no. } & \text { Date } & \text { Time (hr) } & \text { Sodium levels (mEq/L) } \\ 1 & 14 / 11 / 2009 & 2200 & 103.4 \\ 2 & 15 / 11 / 2009 & 0600 & 107.3 \\ 3 & 15 / 11 / 2009 & 1400 & 110.4 \\ 4 & 15 / 11 / 2009 & 2200 & 114.0 \\ 5 & 16 / 11 / 2009 & 0600 & 117.0 \\ 6 & 16 / 11 / 2009 & 2200 & 124.0 \\ 7 & 17 / 11 / 2009 & 1000 & 130.0\end{array}$

in porphyric crisis with severe hyponatraemia and the syndrome of inappropriate antidiuretic hormone secretion (SIADH). Management continued with $3 \% \mathrm{NaCl}$, ventilatory support, and carbepenem group of antibiotic. ABG normalised after 24 hours and serial sodium levels improved (Table 1). The patient was extubated successfully after 24 hours. Computerised tomography scan brain at this stage revealed diffuse cerebral oedema. Urine was sent for porphobilinogen test and the report received on 17 November 2009 was positive. Final diagnosis was a case of an AIP in porphyric crisis with severe hyponatraemia and SIADH. Patient recovered completely without any neurological deficit.

\section{DISCUSSION}

Acute intermittent porphyria is an autosomal dominant disorder resulting from partial deficiency of porphobilinogen deaminase (PBGD) activity, the third enzyme in the pathway of heme synthesis. Acute intermittent porphyria is a rare condition with reported incidence of 1-2 per 100,000 in Europe. ${ }^{1}$ However, its true incidence in Indian subcontinent is unknown.

Attacks are usually precipitated by endogenous sex hormones, dietary restriction, alcoholic drinks, severe infection, fever, stress, or administration of drugs. ${ }^{2}$ In our case, it was probably precipitated by infection, fever and administration of drugs. Hyponatraemia, found in approximately $20 \%$ of patients with symptomatic AIP, is generally due to SIADH. ${ }^{3}$ Seizures in AIP can be due to neurological effects or hyponatraemia due to SIADH. Seizures are difficult to manage as most of antiepileptic drugs are contraindicated in AIP. Bromides which are safe are not available. ${ }^{4}$ Seizures may be seen in 3-15\% of patients with hepatic porphyria. During the seizure episode, the patient may be given benzodiazepines, which in low doses are said to be safe in AIP. ${ }^{5}$

Except for gabapentin and vigabatrin, almost all other antiepileptic drugs can exacerbate porphyric crisis. ${ }^{6}$ In our case, seizures were due to SIADH which was managed with benzodiazepines. Syndrome of inappropriate antidiuretic hormone secretion is a rare manifestation of AIP and very few cases have been reported in literature. ${ }^{7,8}$

Our patient presented with clinical features of pain abdomen, gastrointestinal symptoms, psychiatric manifestations and severe symptomatic hyponatraemia leading to generalised seizures due to SIADH. He was evaluated at number of gastrointestinal centres for pain in abdomen and diagnosis could not be established. He also visited psychiatrist for recurrent pain in abdomen. Diagnosis of AIP was confirmed by raised urinary porphobilinogen (PBG) levels and SIADH was diagnosed by severe euvolemic hyponatraemia with urinary sodium of $163 \mathrm{mmol} / \mathrm{L}$. Heme arginate could not be given as it was not available; however, the patient made complete recovery with symptomatic management without any neurological deficit.

\section{CONFLICTS OF INTEREST}

None identified.

\section{REFERENCES}

1. Anderson KE, Bloomer JR, Bonkovsky HL, Kushner JP, Pierach CA. Recommendations for the diagnosis and treatment of the acute porphyrias. Ann Intern Med 2005;142:439-450.

2. Kochar SK, Mahajan M, Gupta RP, et al. Acute attacks of AIP (acute intermittent porphyria) with severe vivax malaria associated with convulsions: a case report. J Vector Borne Dis 2009;46:307-309.

3. Ashawesh K, Jones MK. Acute intermittent porphyria associated with inappropriate $\mathrm{ADH}$ secretion in a hyperthyroid patient. Internet J Endocrinol 2006;2.

4. Bonkowsky HL, Sinclair PR, Emery S, Sinclair JF. Seizure management in acute hepatic porphyria. Neurology 1980;30:588.

5. Bylesjo I, Forsgren L, Lithner F, Boman K. Epidemiology and clinical characteristics of seizures in patients with acute intermittent porphyria. Epilepsia 1996;37:230-235.

6. Adel A Al Jishi, Sreekantaswamy. Fatal neurological complications of acute intermittent porphyria. Bahrain Med Bull 2004;26:67-69.

7. Usalan C, Erdem Y, Altun B, et al. Severe hyponatremia due to SIADH provoked by acute intermittent porphyria. Clin Nephrol 1996; 45:418.

8. Chogle AR, Shetty RN, Joshi VR, Shanbhag VV. Acute intermittent porphyria with the syndrome of inappropriate ADH secretion (SIADH): a report of two cases. J Assoc Physicians India 1980;28:379-382. 